\title{
A TWO-LEVEL NON-INTRUSIVE COUPLING METHOD FOR FATIGUE ANALYSIS
}

\author{
Yanjie Liu, ${ }^{1, *}$, Qin Sun $^{2}$ \\ ${ }^{1}$ AVIC the First Aircraft Institute, Xi'an, China \\ ${ }^{2}$ Northwestern Polytechnical University, School of Aeronautics, Xi'an, China
}

\begin{abstract}
In the present paper, based on the non-intrusive coupling concept and the continuum damage mechanics, a two-level non-intrusive coupling strategy for fatigue analysis procedure is developed to efficiently and accurately predict the fatigue damage of critical details in a large structure. Coupled multi-scale models including the global structure (coarse mesh) and local details (fine mesh) are involved to evaluate fatigue damage in local details and their effect on the global structure. In this method, the fatigue analysis in critical region is divided into two levels the local boundary condition update and the local fatigue analysis. In the first level, the external cyclic load is transferred from global scale to the local scale. And in the second level, the local fatigue analysis driven by the local cyclic boundary is carried out. The computational procedure of this two-level analysis can be fully coupled, semi-coupled or uncoupled according to different scenarios. Furthermore, the low-cycle fatigue damage is studied and the corresponding fatigue life predictions are simulated on a plate with a central circular hole under cyclic load. The results reveal that the present method greatly improves the numerical efficiency and accords well with the traditional FE analysis with global fine mesh.
\end{abstract}

\section{Introduction}

With the rapid development of large structures applied in aerospace and civil engineering, it is significant to maintain structure integrity and safety under various loading environments during service. Since most load-bearing components in engineering are subjected to cyclic loads, fatigue failure is one of the most significant threats to the structure, thus the prediction of fatigue life becomes critically important. In the past two decades, a vast amount of researches on the mechanism of fatigue damage and the numerical prediction of fatigue life have been conducted [1-4]. However, many obstacles still lead to serious limitations on the practical application of fatigue life prediction of large structure. One of the most difficult problems is the compromise between accuracy and efficiency in the fatigue damage analysis of hot-spots (e.g. bolts, rivets and holes) in a large structure, especially, involving complex inelastic deformation. Actually, the dilemma of accuracy and efficiency is caused by the fact that the FE discrete scales adopted of global and local behaviours are largely different [5-8].

\footnotetext{
* Corresponding author: lyanjie1988@163.com
} 
In order to overcome these barriers, the non-intrusive coupling method [9-13], further developed by Liu [12], provides a novel and feasible way to deal with the large structural analysis with local nonlinearities. It shares the following major advantages: (1) coupled multi-scale model, i.e., it couples a global linear model (structure scale) and several local complex non-linear models where possible damage is involved (material scale) in an iterative way; (2) non-intrusiveness, i.e., it is non-intrusive with the FE solver and can be implemented into commercial code to perform large scale analysis; (3) independence of global analysis, i.e., it avoids modifying the global linear model during analysis, which means the global model will be assembled on the full structure only once and never changed, whereas the local model can be modified and performed as many times as necessary; (4) parallel computation, i.e., the local models can be individually analyzed through parallel computing for the data exchange happens between local interfaces and global interface and there is no need to exchange information between local models. Generally speaking, it realizes to integrate the cycle of CAD modeling, FE analysis and modifications of local details with the least effort to only modify the local model.

In the present paper, within the non-intrusive coupling framework [9, 12], a two-level local fatigue analysis method is provided to efficiently and accurately predict fatigue life of structure with local nonlinearity. This method decomposes the fatigue analysis on an original global structure into two levels, i.e. the local boundary condition update and the local fatigue analysis. In contrast with the traditional FE method with global fine mesh, the proposed two-level method puts more concentrations on local nonlinear analysis, thus improving the numerical efficiency.

\section{Two-level non-intrusive coupling method for fatigue analysis}

\subsection{Non-intrusive coupling strategy}

The non-intrusive coupling method [9] is proposed to overcome the disadvantages of traditional FE global/local method in which the local nonlinear analysis is only affected by global linear analysis through one-way computation while in turn the influence of local nonlinearity with complex details on the global scale is never considered. Therefore, the traditional FE global/local method is not suitable to accurately capture displacement field along the global and local interface when nonlinearity occurs in local region.

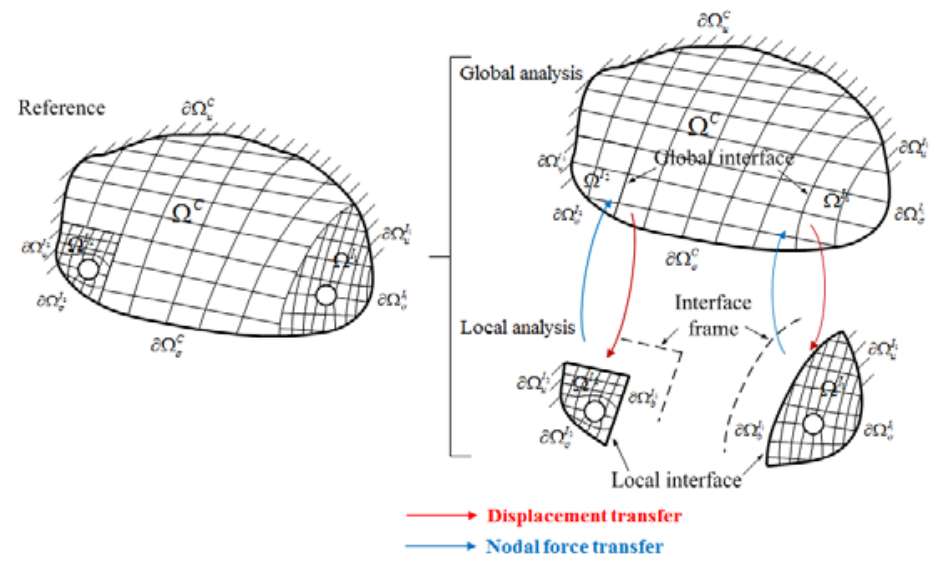

Fig. 1. Definition of the global model, the local model as well as the reference model. 
In order to implement the non-intrusive coupling algorithm into the FE environments, two FE models, the global model (coarse mesh) and the local model (fine mesh), should be defined. The global model with coarse mesh represents the whole structure $\Omega$ (including the local interested regions $\Omega^{I}$ and the complementary region $\Omega^{C}$ ) without details of local geometry and is assumed linear elastic, whereas the local model with fine mesh represents $\Omega^{I}$ alone with details of local geometry and is assumed to be nonlinear (Fig. 1). The iterative algorithm finally converges to a reference solution (the reference model is depicted in the Fig. 1) of the global coarse model with its inaccurate local parts replaced by the nonlinear solutions of local fine models. The detailed procedures with the implementations are introduced in [12].

\subsection{Two-level fatigue life analysis procedure}

In this section a two-level non-intrusive fatigue analysis method is proposed in which the analysis of a large structure with local fatigue damage in hot-spots under the cyclic load is divided into two levels, i.e. (1) scaling cyclic load from global scale to local scale and (2) local fatigue analysis under local cyclic load. The procedure of the two-level fatigue analysis is as follows:

Step 1 (level 1): non-intrusive coupling analysis under the reference load. Choose the amplitude of external cyclic stress or displacement as the reference load $L_{\text {reference }}$ and conduct non-intrusive coupling analysis under reference load till the solution converges. After convergence, the results of interface displacement $u_{\text {reference, } \Gamma}^{L}$ of local model can be obtained and referred to as the magnitude of local cyclic load for the local fatigue analysis.

Step 2 (level 1): Determine local cyclic load as boundary condition. Then, with the reference load $L_{\text {reference }}$ and the external cyclic load $L$, the local cyclic displacement $u_{\Gamma}^{L}$, i.e. local cyclic boundary condition, can be determined,

(1)

$$
u_{\Gamma}^{L}(t)=r_{\text {local }}(t) \cdot u_{\text {reference, },}^{L},
$$

where $r_{\text {local }}$ is coefficient of local cycles, which is determined by

$$
r_{\text {local }}(t)=\frac{L(t)}{L_{\text {reference }}} \text {. }
$$

Step 3 (level 2): Fatigue analysis in the local model. The above steps are referred to as the first level to obtain local cyclic load, i.e. the cyclic displacement on the boundary of local model. Then, with the given fatigue damage law, fatigue analysis can be conducted in the local model driven by the local cyclic load $u_{\Gamma}^{L}$.

Step 4 (level 2): Local model update and global correction for interface solution. Concerning the coupling effect of damage on the constitutive relations, the material degradation in the local model caused by damage accumulation makes local region "soft", which in turn changes the interface solution in the global scale. Therefore, the local model update strategy is needed to correct the global interface solution.

The fully coupled update requires the damage of local model to be updated every increment in local analysis, which is very time-consuming and unnecessary. Most of time the damage update of local model can be conducted every several blocks of cycles, which forms the semi-coupled method. Moreover, if the local region is large enough, there is even no need to update the local model during the local fatigue analysis since the accumulated damage in the hot-spot has almost no influence on the far away local boundary, which forms the uncoupled method. 
The flow chart of this two-level analysis is shown in the Fig. 2.

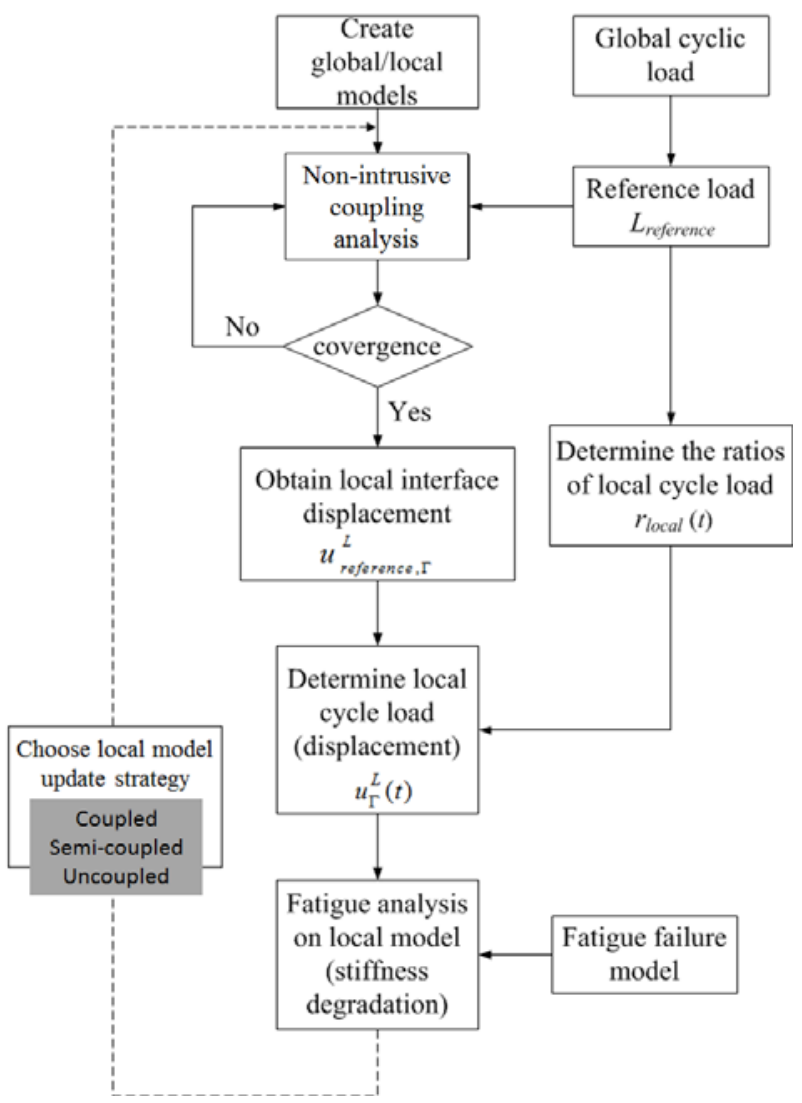

Fig. 2. Flow chart of the two-level non-intrusive coupling strategy for fatigue analysis.

\section{Damage coupled model for low-cycle fatigue}

The proposed method in section 2 is independent of the fatigue life criteria chosen in the local fatigue analysis. In the present paper a continuum damage mechanics (CDM) based fatigue damage law is adopted for demonstration.

Base on the CDM, a damage law has been proposed by Lemaitre [2], in which the damage growth was governed by plasticity and enhanced by the elastic energy. For low cycle fatigue, the basic damage evolution can be written as

$$
d D=\left\{\begin{array}{l}
\left(\frac{Y}{S}\right)^{s} d p, \quad p \geq p_{D}, \quad Y=\frac{\sigma_{e q}^{2} R_{v}}{2 E(1-D)^{2}}, \\
0, \quad p<p_{D}
\end{array}\right.
$$

(3)

where $D$ is the damage value, $p$ is the accumulated plastic strain, $S$ and $s$ are damage parameters, $Y$ is the elastic energy density, in which $R_{v}$ is the stress triaxiality functionThe damage initiates only if the accumulated plastic strain $\mathrm{p}$ reaches the critical value $p_{D}$.

However, if multiple cyclic loads are employed, the $p_{D}$ is different for each cyclic load, and the $N_{0}$ is determined if the summations of accumulated equivalent plastic strain at each cycle reaches the condition of damage initiation, 


$$
\sum_{i=1}^{N_{0}} \frac{\Delta p_{i}}{p_{D}^{i}}=1
$$

(4)

where the $\Delta p_{i}$ is the corresponding increment of equivalent plastic strain at the $i$ th cycle of loading. The total fatigue life (number of cycles to failure) $N_{R}$ is the summation of $N_{0}$ and $N_{D}$

$$
N_{R}=N_{0}+N_{D}
$$

\section{Two-level numerical simulation of low-cycle fatigue}

In this section, fatigue life prediction of a plate structure with a center circular hole is performed using the proposed two-level analysis. The implementation of the proposed two-level non-intrusive coupling method for fatigue analysis is based on the commercial solver ABAQUS and the developed programs via the user interfaces provided by ABAQUS (i.e. Python script to control iterations and user's subroutine UMAT to code numerical fatigue damage law).

\subsection{FE Modeling}

In the present two-level method, the FE modelling of the studied plate with a central hole is build up by a global model and a local model. The global model representing a component part of a large structure is built with $2 \mathrm{D}$ shell elements in structural scale and the local model represents the local details are recovered with 3D solid mesh [13] (Fig. 3). Note that the global and local meshes are incompatible along the interface. In numerical simulations, the constant amplitude cyclic loading is applied with maximum displacement $u$ $=0.45 \mathrm{~mm}$. The material constants of elasto-plastic constitutive and fatigue damage law [2] are listed in Table 1.

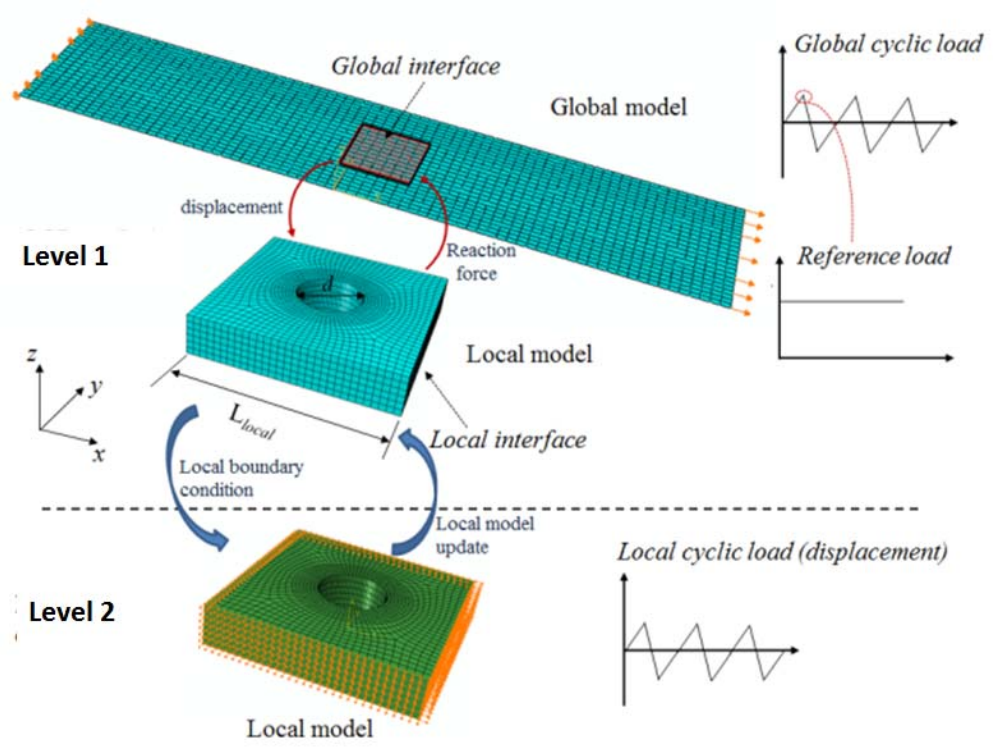


Fig. 3. Global and local models used in non-intrusive coupling analysis and fatigue life analysis.

Table 1. Material constants of AL7050.

\begin{tabular}{cccc}
\hline Young's modulus & Poisson's ratio & Initial yield stress & Tangent modulus \\
\hline $69 \mathrm{GPa}$ & 0.33 & $434 \mathrm{MPa}$ & $800 \mathrm{MPa}$ \\
\hline$\cdot p d$ & $\cdot u$ & $S$ & $s$ \\
\hline 0.12 & $530 \mathrm{MPa}$ & 3.724 & 2.273 \\
\hline
\end{tabular}

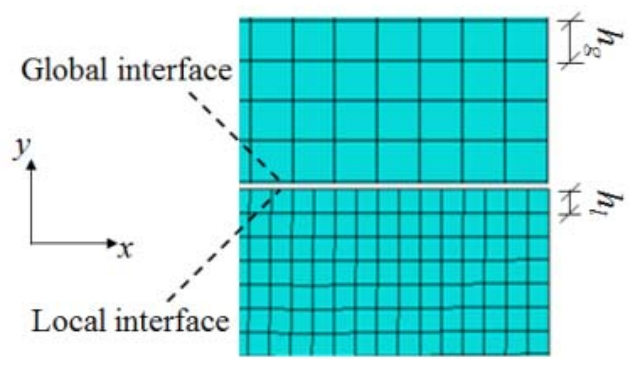

Fig. 4. The ratio of element sizes along the interface.

Different sizes of local region (i.e. $L_{\text {local }}=3 \mathrm{~d}, 4 \mathrm{~d}$ and $5 \mathrm{~d}$ in Fig. 3.) and element size ratios at the interface (i.e. $h_{g} / h_{l}=3,4$ and 5 in Fig. 4 ) are investigated in this work.

In order to verify the accuracy of the present two-level method, a FE model of entire structure with local details and the same fine mesh density as local model is built and defined as the FE reference model. The traditional fatigue analysis is performed on this FE reference model and the result is defined as the reference result.

\subsection{Results and discussions}

The numerical results of the predicted fatigue life for coupled, semi-coupled and uncoupled procedures are shown in Table 2. It can be seen that the numerical results of predamage life $N_{0}$ predicted by coupled, semi-coupled and uncoupled procedures are the same, which are only influenced by the local size $L_{\text {local }}$ and interface element ratio $h_{g} / h_{l}$. In other words, it revealed that the accuracy of $N_{0}$ is only associated with the accuracy of interface solution (stress and plastic strain) from non-intrusive coupling analysis.

However, numerical results show significant differences in predictions of damageaccumulated life $N_{D}$ for coupled, semi-coupled and uncoupled methods. For all the three update procedures, the error of $N_{D}$ increases along with the increasing of interface element ratio $h_{g} / h_{l}$. Moreover, for coupled procedure, the local region size $L_{\text {local }}$ has almost no influence on $N_{D}$. Especially, for cases of $L_{\text {local }}=4 \mathrm{~d}$ and $5 \mathrm{~d}$, the result of damage-accumulated life $N_{D}$ from coupled method are the same with FE reference results for different interface element ratio $h_{g} / h_{l}$ and the error of total fatigue life only comes from the prediction of predamage life $N_{0}$. While, for uncoupled procedure, the $N_{D}$ is very sensitive to the local region size $L_{\text {local }}$, suggesting that the error of $N_{D}$ decreases along with the increasing of local size $L_{\text {local }}$. This sensitivity of local size $L_{\text {local }}$ can be largely reduced through semi-coupled procedure. 
Table 2. The predicted fatigue life and the accuracy of local analysis.

\begin{tabular}{|c|c|c|c|c|c|c|c|}
\hline \multirow[b]{2}{*}{ No. } & & \multicolumn{2}{|c|}{$h_{g} / h_{l}=3$} & \multicolumn{2}{|c|}{$h_{g} / h_{l}=4$} & \multicolumn{2}{|c|}{$h_{g} / h_{l}=5$} \\
\hline & & $\begin{array}{c}N_{D} \\
\left(N_{0}\right)\end{array}$ & Error & $\begin{array}{c}N_{D} \\
\left(N_{0}\right) \\
\end{array}$ & Error & $\begin{array}{c}N_{D} \\
\left(N_{0}\right) \\
\end{array}$ & Error \\
\hline \multirow{3}{*}{$\begin{array}{c}\mathrm{L}_{\text {local }}= \\
\quad 3 d\end{array}$} & Coupled & $\begin{array}{c}210 \\
(345)\end{array}$ & $\begin{array}{c}0.0 \% \\
(0.0 \%)\end{array}$ & $\begin{array}{c}210 \\
(345)\end{array}$ & $\begin{array}{r}0.0 \% \\
(0.0 \%)\end{array}$ & $\begin{array}{c}220 \\
(355)\end{array}$ & $\begin{array}{c}4.8 \% \\
(2.9 \%)\end{array}$ \\
\hline & $\begin{array}{l}\text { Semi- } \\
\text { coupled }\end{array}$ & $\begin{array}{c}215 \\
(345)\end{array}$ & $\begin{array}{c}0.0 \% \\
(0.0 \%)\end{array}$ & $\begin{array}{c}220 \\
(345)\end{array}$ & $\begin{array}{c}4.8 \% \\
(0.0 \%)\end{array}$ & $\begin{array}{c}220 \\
(355)\end{array}$ & $\begin{array}{c}4.8 \% \\
(2.9 \%)\end{array}$ \\
\hline & $\begin{array}{c}\text { Un- } \\
\text { coupled }\end{array}$ & $\begin{array}{c}225 \\
(345)\end{array}$ & $\begin{array}{l}7.1 \% \\
(0.0 \%)\end{array}$ & $\begin{array}{c}230 \\
(345)\end{array}$ & $\begin{array}{c}9.5 \% \\
(0.0 \%)\end{array}$ & $\begin{array}{c}230 \\
(355)\end{array}$ & $\begin{array}{c}9.5 \% \\
(2.9 \%)\end{array}$ \\
\hline \multirow{3}{*}{$\begin{array}{c}\mathrm{L}_{\text {local }}= \\
\quad 4 d\end{array}$} & Coupled & $\begin{array}{c}210 \\
(345)\end{array}$ & $\begin{array}{c}0.0 \% \\
(0.0 \%)\end{array}$ & $\begin{array}{c}210 \\
(345)\end{array}$ & $\begin{array}{c}0.0 \% \\
(0.0 \%)\end{array}$ & $\begin{array}{c}210 \\
(350)\end{array}$ & $\begin{array}{c}0.0 \% \\
(1.4 \%)\end{array}$ \\
\hline & $\begin{array}{l}\text { Semi- } \\
\text { coupled }\end{array}$ & $\begin{array}{c}210 \\
(345)\end{array}$ & $\begin{array}{c}0.0 \% \\
(0.0 \%)\end{array}$ & $\begin{array}{c}205 \\
(345)\end{array}$ & $\begin{array}{r}2.4 \% \\
(0.0 \%)\end{array}$ & $\begin{array}{c}200 \\
(350)\end{array}$ & $\begin{array}{l}-4.8 \% \\
(1.4 \%)\end{array}$ \\
\hline & $\begin{array}{c}\text { Un- } \\
\text { coupled }\end{array}$ & $\begin{array}{c}195 \\
(345)\end{array}$ & $\begin{array}{l}-7.1 \% \\
(0.0 \%)\end{array}$ & $\begin{array}{c}195 \\
(345)\end{array}$ & $\begin{array}{l}-7.1 \% \\
(0.0 \%)\end{array}$ & $\begin{array}{c}200 \\
(350)\end{array}$ & $\begin{array}{l}-4.8 \% \\
(1.4 \%)\end{array}$ \\
\hline \multirow{3}{*}{$\begin{array}{c}\mathrm{L}_{\text {local }}= \\
5 d\end{array}$} & Coupled & $\begin{array}{c}210 \\
(345)\end{array}$ & $\begin{array}{c}0.0 \% \\
(0.0 \%)\end{array}$ & $\begin{array}{c}210 \\
(345)\end{array}$ & $\begin{array}{c}0.0 \% \\
(0.0 \%)\end{array}$ & $\begin{array}{c}210 \\
(345)\end{array}$ & $\begin{array}{c}0.0 \% \\
(0.0 \%)\end{array}$ \\
\hline & $\begin{array}{l}\text { Semi- } \\
\text { coupled }\end{array}$ & $\begin{array}{c}210 \\
(345)\end{array}$ & $\begin{array}{c}0.0 \% \\
(0.0 \%)\end{array}$ & $\begin{array}{c}210 \\
(345)\end{array}$ & $\begin{array}{c}0.0 \% \\
(0.0 \%)\end{array}$ & $\begin{array}{c}210 \\
(345)\end{array}$ & $\begin{array}{c}0.0 \% \\
(0.0 \%)\end{array}$ \\
\hline & $\begin{array}{c}\text { Un- } \\
\text { coupled }\end{array}$ & $\begin{array}{c}210 \\
(345)\end{array}$ & $\begin{array}{c}0.0 \% \\
(0.0 \%)\end{array}$ & $\begin{array}{c}210 \\
(345)\end{array}$ & $\begin{array}{c}0.0 \% \\
(0.0 \%)\end{array}$ & $\begin{array}{c}210 \\
(345)\end{array}$ & $\begin{array}{c}0.0 \% \\
(0.0 \%)\end{array}$ \\
\hline Refere & analysis & $N_{D}^{R}=$ & $0 N_{0}^{R}=$ & $J\left(N_{R}\right.$ & $N_{0}^{R}+$ & $=550$ & \\
\hline
\end{tabular}

(a)

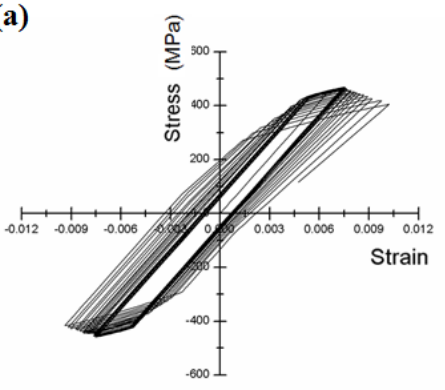

(b)

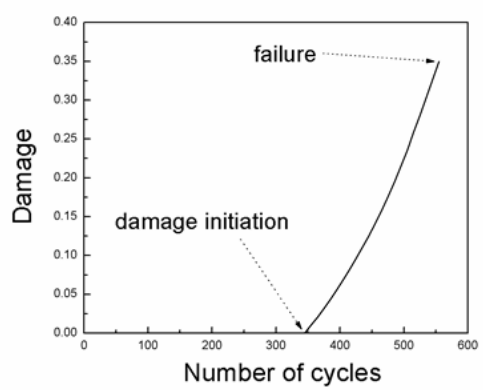

Fig. 5. (a) Stress-strain curve of the critical point at the rim of the hole with damage accumulation.

(b)Damage evolution vs. number of cycles 
The reason of the difference in damage-accumulated life $N_{D}$ prediction for coupled, semi-coupled and uncoupled methods lies in the fact that in coupled procedure the amplitude of local cyclic displacement field evolves along with cycles due to the degradation of local model caused by damage accumulation shown in Fig 5.

Although the semi-coupled and uncoupled procedures are less accurate than the coupled one, the fatigue life predicted by semi-coupled and uncoupled procedures also has a good accuracy compared with reference FE results if the size of local region $L_{\text {local }}$ is appropriated adopted.

\section{Conclusions}

In this paper, a two-level non-intrusive coupling method for fatigue life prediction method is proposed and implemented to the commercial ABAQUS code through the developed user interface provided by ABAQUS. A correct local boundary is obtained in the first level, followed by the local fatigue analysis in the second level. And the method is independent with the fatigue model applied in local analysis. In this paper, three different local model update strategies are given to account for the local material degradation. Numerical simulation shows that the semi-coupled update procedure not only has high efficiency but also achieves good accuracy. Besides, since the global model is not modified in the analysis, this can be helpful for testing various local modification plans in structure design.

\section{REFERENCES}

1. R. Desmorat, A. Kane, M. Seyedi, J. P. Sermage, Eur. J. Mechanics A/Solids 26, 909 (2007)

2. J. Lemaitre, R. Desmorat, Engineering damage mechanics: ductile, creep, fatigue and brittle failures (Springer Science \& Business Media, 2005)

3. X. Q. Peng, L. Geng, W. Liyan, G. R. Liu, K. Y. Lam, Comput. Mech. 21, 253(1998)

4. M. F. Arif, N. Saintier, F. Meraghni, J. Fitoussi, Y. Chemisky, G. Robert, Compos. Part B Eng. 61, 55(2014)

5. Y. J. Liu, Q. Sun, X. L. Fan, T. Suo, Mat. Sci. Eng. A-Struct. 576, 337(2013)

6. J. Rannou, N. Limodin, J. Réthoré, A. Gravouil, W. Ludwig, M. C. Baïetto-Dubourg, J. Y. Buffière, A. Combescure, F. Hild, S. Roux, Comput. Methods Appl. Mech. Eng. 199, 1307(2010)

7. J. Pebrel, C. Rey, P. Gosselet, Int. J. Multiscale Com. 6, 251(2008)

8. Z. X. Li, T. Q. Zhou, T. H. Chan, Y. Yu, Eng. Struct. 29, 1507(2007)

9. L. Gendre, O. Allix, P. Gosselet, F. Comte, Comput. Mech. 44, 233(2009)

10. J. C. Passieux, J. Réthoré, A. Gravouil, M. C. Baietto, Comput Mech 52, 1381(2013)

11. M. Duval, J. C. Passieux, M. Salaün, S. Guinard, Arch. Comput. Methods Eng. 23, 17(2016)

12. Y. J. Liu, Q. Sun, X. L. Fan, Comput. Methods Appl. Mech. Eng. 277, 81(2014)

13. G. Guguin, O. Allix, P. Gosselet, S. Guinard, Int. J. Numer. Methods Eng. 98, 324(2014) 